\title{
Promoting the Advanced Nursing Practice role in Latin America
}

\author{
Silvia Helena De Bortoli Cassiani', Keri Elizabeth Zug" \\ ' Pan American Health Organization / World Health Organization, Regional Advisory on Nursing and \\ Allied Health Personnel, Health Systems and Services. Washington D.C, United States of America. \\ "Pan American Health Organization / World Health Organization, Health Systems and Services. \\ Washington D.C, United States of America.
}

The healthcare environment in Latin America is exceedingly complex. Disparities in access to quality health care in addition to a diverse disease burden of both communicable and non-communicable diseases continue to pose challenges for effective health resource allocation and delivery ${ }^{(1)}$. Universal Health Coverage and Universal Access to Health (UHC/UAH) is an overarching goal of health systems emphasizing broad access to primary health care (PHC). Its implementation realizes the broadly held value that all people and communities should have equitable access to the quality health services they need throughout the life span without financial hardship. In May 2014, the Executive Committee of the Pan American Health Organization (PAHO) proposed the Strategic Plan for Universal Health Coverage (2). PAHO plans to support Ministries of Health in Latin America to incorporate strategic planning for UHC/UAH into countries health system, focusing on quality $\mathrm{PHC}$. A primary consideration of UHC implementation is the composition of human resources for health $(\mathrm{HRH})$, particularly nurses.

Acknowledging the critical importance of $\mathrm{HRH}$ to achieve the goal of UHC/UAH, PAHO previously released a Resolution in September 2013: Human Resources for Health: Increasing Access to Qualified Health Workers in Primary Heath Care-Based Health Systems. The Resolution specifically calls to increase the number of advanced practice nurses (APNs) to support PHC-based health systems ${ }^{(3)}$.

The PAHO Resolution has significant implications for the future of nursing in Latin America.

The nursing profession is continuously evolving to meet global health challenges. Nurses are prepared to respond and manage health throughout the life course. Within the context of primary health care, they can greatly contribute to the reduction of morbidity and mortality often in the most underserved areas. Nurses are frontline professionals who use an integrated and comprehensive approach including health promotion, disease prevention, treatment, rehabilitation and palliative care.

However, while nursing professionals have the capacity to drastically improve and expand the breadth of PHC in Latin America, much must change to meet the challenges of delivering on UHC/UAH.

Nursing personnel in rural areas often provide primary care services to underserved groups, essentially practicing in an advanced practice role, but many lack the formalized skill training, defined role and graduate level education to support this degree of practice independence ${ }^{(4)}$. According to the International Council of Nurses, International Nurse Practitioner/Advanced Practice Nursing Network, APNs are "registered nurses who have acquired the expert knowledge base, complex decision-making skills, and clinical competencies for expanded practice, the characteristics of which are shaped by the context or country in which they are credentialed to practice. A master's level degree is recommended for entry level"(5). Currently in many Latin American countries, necessary advanced practice clinical skills are often sought on the job in response to the health needs of the population served, and not as part of a formal APN program. As such, advanced nursing practice competency and continuing education opportunities need to be accredited and managed by nursing faculty at the university level.

Reforms to the preparation of the nursing workforce in Latin America must occur both externally and internally. Externally, it is critical to develop policy infrastructure to support the nursing profession including regulation, scope of practice, certification, undergraduate and graduate level education, continuing education and salary 
reform. Internally, a shift in attitude must occur within nursing that posits nurses as essential components to the success of primary health care initiatives. Nurses possess a unique practice perspective from that of physicians that prioritizes holistic person-centered care consistent with the nursing model. A common identity and uniting nursing culture must be encouraged among Latin American nurses to support the standardization of the APN role.

In response to the recent Resolution pertaining to $\mathrm{HRH}, \mathrm{PAHO}$ is currently establishing a working plan to support the expansion and professionalization of advanced practice nursing. This plan will identify goals related to education, regulation and scope of practice of the APN role and establish priority objectives to present to the member states. PAHO also seeks to coordinate a webinar conference series to promote discussion of the APN role in Latin America and familiarize Latin American nursing faculty with nursing practice conditions in other regions.

Changing nursing culture and fostering a broader nursing role in Latin America calls for political will for change, which can only be realized through multifaceted support from government officials, policy makers, hospital directors, medical personnel and nursing faculty, in addition to a change in perspective on the part of professional nurses. Through prioritizing the preparation and professionalization of advanced practice nurses in Latin America, they will be well suited to effectively promote and broadly implement UHC, providing quality primary health care where it is most needed.

The author is a staff member of the Pan American Health Organization. The author alone is responsible for the views expressed in this publication, and they do not necessarily represent the decisions or policies of the Pan American Health Organization.

\section{REFERENCES}

1. Musgrove P. Challenges and solutions in health in Latin America [Internet]. 2007 [cited 2014 September 24]. Available from: http://idbdocs.iadb.org/wsdocs/getdocument.aspx?docnum = 1186232

2. Pan American Health Organization. Strategy for Universal Health Coverage. In: 154th Session of the Executive Committee [Internet]. 2014 June 16-20; Washington, US [cited 2014 September 24]. Available from: file:///C:/ Users/Cliente/Downloads/CE154-3-e.pdf

3. Pan American Health Organization. Resolution CD52.R13. Human resources for health: Increasing access to qualified health workers in primary health care-based health systems. In: 52 nd Directing Council: $65^{\text {th }}$ Session of the Regional Committee [Internet]. 2013 Sep-Oct; Washington, US [cited 2014 September 24]. Available from: file://C:/Users/Cliente/Downloads/CD52-R13-e.pdf

4. Nigenda G, Magaña-Valladares L, Cooper K, Ruiz-Larios JA. Recent developments in public health nursing in the Americas. Int J Environ Res Public Health. 2010;7(3):729-50.

5. Nurse Practitioner/Advanced Practice Nurse: definition and characteristics. Nursing Matters [Internet]. 2009 [cited 2014 September 24]. Available from: https://acnp.org.au/sites/default/files/33/definition_of_apn-np.pdf 